\title{
3D spatially-resolved geometrical and functional models of human liver tissue reveal new aspects of NAFLD progression
}

4 Fabián Segovia-Miranda ${ }^{1}$, Hernán Morales-Navarrete ${ }^{1}$, Michael Kücken ${ }^{2}$, Vincent Moser $^{3}$, 5 Sarah Seifert ${ }^{1}$, Urska Repnik ${ }^{1}$, Fabian Rost ${ }^{2,4}$, Alexander Hendriks ${ }^{5}$, Sebastian Hinz ${ }^{5}$, 6 Christoph Röcken ${ }^{5}$, Dieter Lüthjohann ${ }^{6}$, Yannis Kalaidzidis ${ }^{1}$, Clemens Schafmayer ${ }^{5}$, Lutz 7 Brusch $^{2}$, Jochen Hampe ${ }^{3}$ and Marino Zerial ${ }^{1 *}$.

$10{ }^{1}$ Max Planck Institute of Molecular Cell Biology and Genetics, Dresden, Germany.

$11{ }^{2}$ Center for Information Services and High Performance Computing, Technische Universität 12 Dresden, Dresden, Germany.

$13{ }^{3}$ Medical Department 1, University Hospital of the Technical University Dresden, Dresden, 14 Germany.

$15{ }^{4}$ Max Planck Institute for the Physics of Complex Systems, Dresden, Germany.

16 [ University Hospital Schleswig-Holstein, Kiel, Germany.

$17{ }^{6}$ Institute of Clinical Chemistry and Clinical Pharmacology, University of Bonn, Bonn, 18 Germany.

19 


\section{Abstract}

26 Early disease diagnosis is key for the effective treatment of diseases. It relies on the 27 identification of biomarkers and morphological inspection of organs and tissues. 28 Histopathological analysis of human biopsies is the gold standard to diagnose tissue 29 alterations. However, this approach has low resolution and overlooks 3D structural changes 30 that are consequence of functional alterations. Here, we applied multiphoton imaging, 3D 31 digital reconstructions and computational simulations to generate spatially-resolved 32 geometrical and functional models of human liver tissue at different stages of non-alcoholic 33 fatty liver disease (NAFLD). We identified a set of new morphometric cellular parameters 34 correlated with disease progression. Moreover, we found profound topological defects in the $353 \mathrm{D}$ bile canaliculi (BC) network. Personalized biliary fluid dynamic simulations predicted an 36 increased pericentral biliary pressure and zonated cholestasis, consistent with elevated 37 cholestatic biomarkers in patients' sera. Our spatially-resolved models of human liver tissue 38 can contribute to high-definition medicine by identifying quantitative multi-parametric cellular 39 and tissue signatures to define disease progression and provide new insights into NAFLD 40 pathophysiology. 


\section{Introduction}

High definition medicine is emerging as an integrated approach to profile and restore the health of an individual using a pipeline of multi-parametric analytical and therapeutic technologies ${ }^{1}$. High-definition medicine relies on large data sets, e.g. genomics, metabolomics, to characterize human health at the molecular level. It also relies on imaging, image analysis and computational modelling approaches to identify structural and functional abnormalities in organs and tissues associated with a disease state. Histology has been classically used to characterize tissue structure and remains the method of choice to describe and monitor a large variety of pathologies ${ }^{2}$. However, this technique has several disadvantages, e.g. it is subjective (depends on the pathologist's skills), is often semiquantitative and provides only two-dimensional (2D) information, i.e. does not account for the three-dimensional (3D) complexity of tissues ${ }^{3}$. In recent years, an increasing number of studies have highlighted the importance of considering $3 \mathrm{D}$ information for the histopathological examination of tissues ${ }^{4-7}$. This is particularly crucial for the analysis of 3D structures. The liver is a pertinent example of an organ with a complex 3D tissue organization $^{8}$. It consists of functional units, the liver lobule ${ }^{9,10}$, containing two intertwined networks, the sinusoids for blood flow and the bile canaliculi (BC) for bile secretion and flux ${ }^{9}$.

59 Sinusoids and BC run antiparallel along the central vein (CV)-portal vein (PV) axis. The

60 hepatocytes are the major parenchymal cells and display a peculiar and unique type of cell

61 polarity distinct from that of simple epithelia ${ }^{11}$. Whereas in epithelia all cells share the same orientation with their apical surface facing the lumen of the organ, hepatocytes are sandwiched between the sinusoidal endothelial cells and share the apical surface with multiple neighbouring hepatocytes to form a 3D BC network ${ }^{12,13}$. Such an architecture makes it difficult to grasp the 3D organization of cells and tissue from 2D histological sections.

66 Recent advances in tissue staining, optical clearing and multi-photon microscopy allow 67 imaging thick sections of tissues such that 3D information can be captured ${ }^{14,15}$. Computer 68 software process the images to generate 3D digital reconstructions of tissues, i.e. 69 geometrical models ${ }^{8}$, with single-cell resolution. These provide a detailed quantitative 70 description of the different cells and structures forming the tissue. The geometrical 71 information extracted from the 3D reconstruction can also be used to generate predictive 72 models of tissue function e.g. biliary fluid dynamic ${ }^{16}$, thus gaining novel insights into liver tissue organization and function. Thereby, geometrical models can be used to quantitatively 
74 describe the tissue architecture and function, improving our understanding of liver biology

75 and pathobiology.

76 Non-alcoholic fatty liver disease (NAFLD), defined as an accumulation of

77 triglycerides and lipid droplets (LD) in the liver in absence of alcohol intake (infiltration in >

$785 \%$ of hepatocytes), is rising to the most common chronic liver disease worldwide ${ }^{17}$. NAFLD

79 includes a spectrum of liver diseases, ranging from simple steatosis to non-alcoholic

80 steatohepatitis (NASH) ${ }^{17}$. Whereas steatosis is considered as a "non-progressive" status of

81 the disease, NASH has the potential to progress to more severe stages, such as cirrhosis

82 or hepatocellular carcinoma, leading eventually to liver failure and transplantation ${ }^{3,17}$. Thus,

83 the understanding of the transition from steatosis to NASH as a disease-defining moment

84 for NAFLD prognosis is key to a deeper understanding of disease pathophysiology. Liver

85 biopsy and histological inspection of thin tissue slices $(<10 \mu \mathrm{m})$ constitute the current gold

86 standard for the diagnosis of steatosis and $\mathrm{NASH}^{2,17,18}$. Unfortunately, due to the limitations

87 in providing $3 \mathrm{D}$ information, alterations in $3 \mathrm{D}$ tissue structures such as $B C^{3,17}$, have been

88 overlooked. Therefore, new approaches are required to gain a more complete

89 understanding of NAFLD establishment and its progression to NASH. In this study, we

90 generated 3D spatially resolved geometrical and functional models of human liver tissue for

91 different stages of NAFLD to contribute to a high definition medical diagnosis of disease

92 establishment and progression. 


\section{Tri-dimensional geometrical models of human liver tissue}

To identify parameters that can quantitatively discriminate the transition from simple steatosis to early $\mathrm{NASH}$ (eNASH) we stained, imaged and digitally reconstructed human

98 liver tissue in 3D. We focused on cell and nuclear morphology, LD, and tissue features such

99 as BC and sinusoidal networks, and their spatial distribution along the CV-PV axis. For this

100 we tested 26 antibodies combinatorially with dyes and antigen retrieval protocols

101 (Supplementary Table 2). Our final pipeline (see Methods for details) includes the following 102 steps. First, $100 \mu \mathrm{m}$ liver sections were heated and antigen retrieved using citric acid buffer 103 (CAAR). Second, we stained for BC (CD13), sinusoids (fibronectin), nuclei (DAPI), lipid 104 droplets LD (BODIPY) and cell borders (LDLR), optically cleared with SeeDB ${ }^{19}$ and imaged 105 at high resolution using multiphoton microscopy $(0.3 \mu \mathrm{m} \times 0.3 \mu \mathrm{m} \times 0.3 \mu \mathrm{m}$ per voxel) 106 (Extended Data Fig. 1). Because this protocol did not provide a good cell border staining of 107 the pericentral hepatocytes in STEA and eNASH, for cell-based measurements (see below 108 Fig. 3), we used an alternative protocol without antigen retrieval enabling the staining of 109 sinusoids (fibronectin), nuclei (DAPI), LD (BODIPY) and cell borders (phalloidin). We applied 110 this pipeline to biopsies from twenty-two patients classified into four groups: normal control $111(\mathrm{NC}, \mathrm{n}=6)$, healthy obese $(\mathrm{HO}, \mathrm{n}=4)$, steatosis (STEA, $\mathrm{n}=5$ ) and early NASH (eNASH, $\mathrm{n}$ $112=7$ ). The demographic, clinical and histological details of the samples are summarized in 113 the Supplementary Table 1. All images cover one complete CV-PV axis within a liver lobule. 114 Finally, we reconstructed the various stained components of the tissue using our open115 source software Motion Tracking (http://motiontracking.mpi-cbg.de) as described ${ }^{8}$ (Fig. 1 116 and Supplementary Video 1-2). The generation of geometrical models constituted the basis 117 for the quantitative and structural characterization of the different components forming the 118 liver tissue in the NAFLD biopsies.

\section{Nuclear-based analysis of NAFLD progression}

We first quantified properties of hepatocytes nuclei, such as cell nuclearity and 121 ploidy, since hepatocytes are heterogenous in both mouse and human ${ }^{8,20}$. We quantified 122 nuclear vacuolization/glycogenation given it is a common histological characteristic in 123 NAFLD linked to insulin resistance ${ }^{21,22}$. Finally, we measured nuclear texture homogeneity, 124 a feature associated with various pathological conditions, such as cancer, inflammation, 
125 cardiomyopathy, etc ${ }^{23-26}$, with methylation and acetylation status ${ }^{27}$ and, more recently, with

126 transcriptional activity ${ }^{28}$. We neither observed differences in the proportion of

127 mono/binuclear cells nor in the ploidy between the groups (Extended Data Fig. 2a and b).

128 The average values of several nuclear features showed only modest variations Extended

129 Data Fig. 2). However, many functional and morphological features of the liver change along 130 the CV-PV axis, such as metabolic zonation ${ }^{29,30}$, ploidy ${ }^{8}$, cell volume ${ }^{8}, \mathrm{BC}^{16}$. Therefore, to

131 account for potential morphological heterogeneities along the CV-PV axis, we

132 computationally divided this axis into ten equidistant zones (Extended Data Fig. 2c)

133 (Methods). We found major differences in nuclear elongation around the CV and

134 vacuolization around the PV between the different groups (Extended Data Fig. 2d,e).

135 Moreover, we identified zonated and progressive differences in nuclear homogeneity as

136 disease progresses (Extended Data Fig. 2f-i). Therefore, our analysis reveals that, in spite

137 of modest changes in the average values, the combined zonated values of nuclear

138 vacuolization and texture homogeneity allow discriminating between all four patient groups.

\section{Morphometric parameters of LD correlate with disease progression}

The finding that quantitative spatially-resolved analysis of nuclear parameters can

141 reveal changes that are not evident in an average estimate prompted us to re-evaluate the 142 morphometric characterization of LD. Even though LD are the most typical hallmark of 143 NAFLD, a detailed quantitative description of their size and their spatial localization within 144 the liver lobule has not been achieved yet. Contrary to traditional histology ${ }^{3}$, immunostaining 145 of thick tissue sections preserved most of the LD (Fig 2a). In agreement with the 146 histopathological description of NAFLD ${ }^{3,17}$, a major increase in LD was observed in STEA 147 and eNASH, which were concentrated between the second and the fifth zones (Fig. 2b). 148 However, the LD occupy a higher volume of the tissue in eNASH than STEA. It is known 149 that the LD can present massive differences in size ${ }^{31,32}$. The LD in the human liver samples 150 ranged from ${ }^{\sim} 1 \mu \mathrm{m}^{3}$ to $20,000 \mu \mathrm{m}^{3}$ (Fig. 2c). To inquire whether differences in LD size may 151 correlate with the disease state, we performed a population analysis based on their volume 152 distributions (Fig. 2c). We defined three sub-populations of LD, namely, small $\left(<14 \mu \mathrm{m}^{3}\right)$, 153 medium $\left(14-400 \mu \mathrm{m}^{3}\right)$ and large $\left(>400 \mu \mathrm{m}^{3}\right)$ ones (Fig. $\left.2 \mathrm{~d}-\mathrm{f}\right)$. Strikingly, we found that the 154 three sub-populations varied between disease conditions. Whereas small LD were evenly 155 distributed along the CV-PV in all conditions, large LD were highly enriched towards the 156 pericentral zone in STEA and eNASH, occupying up to $25 \%$ of the tissue volume (Fig. 2f). 
157 Most importantly however, medium LD were mostly present in eNASH and spanning the

158 entire liver lobule (Fig. 2e), hence contributing to discriminate between STEA and eNASH

159 in the periportal area (Fig. 2e).

The zonated increase in LD size during disease progression is such that some LD in

161 the pericentral zone become even larger than a normal hepatocyte. This leads to global

162 changes in tissue structure. To quantify such changes, we measured the spatial distribution

163 of cell density, cell volume and percentage of cell volume occupied by LD. We found $\sim 50 \%$

164 reduction in the number of hepatocytes located between the CV and the middle zone in

165 STEA and eNASH, when compared with $\mathrm{NC}$ and $\mathrm{HO}$ samples (Fig. 3a). This reduction was

166 compensated by a massive increase in cell volume (Fig. 3b). Hepatocytes were two times

167 larger than the average size (Fig. 3b), reaching values up to up to $\sim 100,000 \mu^{3}$ for STEA

168 and NASH (ten times bigger than a small hepatocyte) (Fig. 3c). A population analysis of the

169 hepatocytes based on their volume revealed a characteristic distribution of different cells

170 populations along the liver lobule (Fig. 3c-f). STEA and eNASH were characterized

171 predominantly by small and large hepatocytes which are anti-correlated along CV-PV axis

172 (Fig. 3d-f). Even though cell density and cell volume were practically indistinguishable

173 between STEA and eNASH, we observed a remarkable phenotype regarding the fraction of

174 cell volume occupied by LD (Fig. 3g-i). In eNASH, hepatocytes accumulated LD even in the

175 periportal zone (Fig. 3h, 3i and Supplementary Video 3), suggesting that LD accumulation

176 progressively extends to the PV as the disease progresses.

Altogether, these data reveal profound quantitative morphological disparities in cell size and LD content along the CV-PV axis between NC, HO, STEA and eNASH. Specifically,

179 the percentage of cell volume occupied by LD in the PV and CV zones can serve to 180 discriminate STEA and eNASH.

\section{Alterations in apical protein trafficking}

The massive presence of LD that occupy a large portion of the cytoplasm raises the question of whether trafficking of proteins to the apical plasma membrane of hepatocytes is

184 affected. We analysed the localization of four apical proteins, aminopeptidase N (CD13), 185 bile salt export pump (BSEP), multidrug resistant-associated protein (MRP2) and 186 dipeptidylpeptidase 4 (DPPIV). CD13, BSEP and MRP2 were correctly localized to the 187 apical membrane in all conditions (Extended Data Fig. 1, 3a-b). DPPIV was enriched on the 188 apical membrane with a small fraction on the basal membrane in NC and HO (Extended 
189 Data Fig. 3c). Strikingly, DPPIV was redistributed to the lateral membrane in pericentral

190 hepatocytes in STEA and eNASH, whereas it retained its normal localization on the

191 periportal zone (Extended Data Fig. 3c). Considering that DPPIV follows the transcytotic

192 route to the apical surface ${ }^{33,34}$ whereas BSEP and MRP2 do not ${ }^{34-36}$, the mislocalization of

193 DPPIV suggests a possible disruption of some stage of endocytosis and transcytosis of this

194 cargo molecule in the pericentral hepatocytes. This supports previous findings regarding the

195 misregulation of membrane protein trafficking in NAFLD ${ }^{37}$ and prompted us to evaluate

196 whether the integrity of the BC could be affected during the disease progression.

197 Bile canaliculi network shows geometrical and topological-zonated defects in NAFLD

198 To determine whether 3D structures such as the BC and sinusoidal networks are 199 affected, we carried out a geometrical and topological characterization of both networks.

200 Even though we observed a slight reduction in the total length of the sinusoidal network in 201 STEA and eNASH (Extended Data Fig. 4e), no major defects in sinusoidal microanatomy 202 were detected (Extended Data Fig. 4a-d, f-g) (volume fraction, radius, branching and 203 connectivity). Next, we analysed the BC network. Contrary to the very packed and 204 homogeneous appearance in NC and HO, the BC in STEA and eNASH displayed clear 205 morphological defects which were more pronounced in the pericentral zone (Fig. 4a, b). A 206 more detailed analysis revealed a sustained increase in BC radius in NASH throughout the 207 CV-PV axis (Fig. 4d). In addition, in both STEA and eNASH, we observed a strong reduction 208 in the total length of the BC towards the pericentral zone (Fig. 4a and f). Other geometrical 209 properties of the BC network, such as volume fraction and junction density were unaffected 210 (Fig. 4c and e).

211 Finally, to investigate the topological properties of the BC network, we performed an 212 analysis of network connectivity (see Methods). Surprisingly, we found a pronounced decay 213 in the connectivity in STEA and eNASH towards the pericentral region (Fig. $4 a, b, g$ and $h$, 214 Supplementary Video 4). One possibility is that the alterations in BC may be the 215 consequence of the spatial constrains arising from the presence of large cells in the tissue 216 (Fig. 3f). To test this, we inspected the connectivity of the sinusoidal network. We found that 217 no defect was observed for the sinusoidal network (Extended Data Fig. 4a, $f$ and $g$ ), 218 supporting the idea that the BC network is specifically affected and not an indirect 219 consequence of spatial constraints. Thus, our data point at specific geometrical and 220 topological alterations in the pericentral BC network in both STEA and eNASH. 
221 Personalised model of bile flow predicts increase in bile pressure in the pericentral 222 zone

The observed alterations of BC network architecture are likely to have consequences

224 for liver tissue function, particularly for bile flow. Clearly, information on bile velocity and

225 pressure in disease conditions could be insightful. However, it is not yet possible to measure

226 bile flow in the human liver at the level of BC as in animal models. We recently developed a

227 computational model of bile fluid dynamics, validated its quantitative predictions in mouse

228 models and demonstrated that bile velocity and bile pressure distributions along the liver

229 lobule strongly depend on BC geometry ${ }^{16}$. However, this model ${ }^{16}$ is not suitable to handle

230 the extreme inhomogeneity of BC density such as the ones encountered in tissue distorted

231 by the presence of large LD (i.e. in STEA and eNASH). Therefore, we addressed this issue

232 and further developed our model in a spatially-resolved fashion (Fig. 5a). Shortly, the refined

233 model is based on conservation of mass for water and osmolytes and Darcy's Law for

234 laminar flow. The proportionality constant in Darcy's Law was derived from the porous media

235 theory. Boundary conditions were set to zero velocity at the outer surface of the central vein,

236 and ambient pressure at the portal outlet. Since we obtained morphometric data for liver

237 tissue from individual patients, we aimed at developing personalised models, i.e.

238 parameterized by individual geometrical measurements $\left(B C\right.$ volume fraction $\varepsilon_{B C}, B C$ radius

$239 r_{\mathrm{BC}}$., fraction of connected BC, canaliculi tortuosity $\tau$, apical surface density $A$, intra-

240 canalicular volume fraction occupied by microvilli 1- $\alpha$ ) (Fig. 4c, 4d, 4g, and Extended Data

241 Fig. 5) and previously reported values (viscosity, permeability and osmolyte secretion rate).

242 No free parameters remained and, hence, no parameter fitting was needed (see Methods

243 for details). Next, we applied this model to predict bile velocity, pressure and solute

244 concentration distributions across the liver lobule for individual patients liver tissue 3D

245 reconstructions from all the four groups.

246 The model predicts bile velocities in the periportal area of about $1.2 \pm 0.4 \mu \mathrm{m} / \mathrm{sec}$ for

247 all patient groups (Fig. 5b-e). Very similar velocities have been reported in mouse ${ }^{16}$.

248 However, the predicted pressure in the pericentral area differed significantly between the

249 patient groups. In the NC and HO groups this pressure was predicted to be lower than 1500

$250 \mathrm{~Pa}$ in all patients (963.2 $\pm 285.2 \mathrm{~Pa}$, mean $\pm \mathrm{SD}$ ) (Fig. 5b-c). This is consistent with the

251 reported maximum biliary secretion pressure of 1,000-1,500 $\mathrm{Pa}$ in the extrahepatic biliary

252 system in rats $^{38}$. In the STEA and eNASH groups, the model predicted an abrupt increase 
253 of bile pressure toward the pericentral zone (Fig. 5d-e). For six STEA and eNASH patients 254 (55\%), the pericentral pressure exceeded 1,500 $\mathrm{Pa}$ and for four patients (36\%) it exceeded 255 twice the maximum pressure predicted for NC (3000 Pa) (Fig. 5d-e). The compensatory 256 effect in bile pressure observed in eNASH is mostly due to the dilation of BC (Fig. 4d). 257 Therefore, our model predicts an increase in pericentral bile pressure in STEA and eNASH 258 conditions ranging from relatively mild to quite severe, depending on the BC geometry of 259 individual patients.

We next set out to validate the model predictions. As it is impossible to measure bile flow and pressure in the human liver, we considered possible consequences of changes in 262 bile pressure. Increased bile pressure is a hallmark of cholestasis ${ }^{39,40}$. Therefore, as readout 263 of increased bile pressure, we analysed the most commonly used cholestatic biomarkers in 264 serum, including bilirubin, gamma glutamyl transpeptidase (GGT), alkaline phosphatase 265 (ALP) and BAs. To increase the statistical power, we analysed additional sera samples for 266 the different groups $(\mathrm{NC}=25, \mathrm{HO}=25, \mathrm{STEA}=24$ and $\mathrm{eNASH}=26$ samples $)$. Whereas 267 we found that both bilirubin and GGT were elevated in STEA and eNASH (Extended Data 268 Fig. 6a-b), we did not detect significant changes in the levels of ALP, total BAs and primary 269 BAs between the groups (Extended Data Fig. 6c, 7b,d). Strikingly, when we analysed the 270 correlation between the predicted pericentral bile pressure and the biomarkers for individual 271 patients from all groups, we found a strong correlation for the majority of the cholestatic 272 biomarkers, with GGT having the strongest correlation (Pearson correlation coefficient ALP= 273 0.473, total BAs 0.505, primary BA 0.518 and GGT 0.680; (Extended Data Fig. 8a-e and 274 Fig. 5f). In contrast, aspartate aminotransferase (AST) and alanine aminotransferase (ALT), 275 biomarkers of hepatocellular liver damage which are not increased in cholestasis, showed 276 no correlation (Extended Data Fig. 8f-g). The presence of pericentral cholestasis was also 277 supported by the increase in the predicted pericentral concentration of BAs (proportional to 278 the lumped concentration of all osmolytes. Extended Data Fig. 9), which in combination with 279 high pressure and low flow velocity, are signs of a defective bile flow. Altogether, our model 280 predicts a significant degree of zonated cholestasis as a new component of the NAFLD 281 pathophysiology. 


\section{Discussion}

High definition medicine provides a novel approach to understand human health of individuals with unprecedented precision ${ }^{1}$. One of its pillars is the combination of image analysis and computational modelling to uncover tissue alterations at different structural and functional levels during disease progression. During the last years, there has been an enormous interest in getting a better understanding of NAFLD establishment and progression due to its growing impact on public health ${ }^{41}$. A lot of attention has been mostly drawn to the role of signalling pathways ${ }^{42-44}$, microbiome ${ }^{45,46}$, metabolism ${ }^{47}$, genetic risk factors ${ }^{48}$, BAs ${ }^{44}$, etc. However, a major challenge is to understand how the molecular alterations detected are expression of the organ dysfunction, manifested as morphological and functional alterations of cells and tissue architecture.

The classical histological analysis has provided insights into fundamental aspects of NAFLD. However, a quantitative description of the 3D tissue morphology is indispensable, particularly for the liver which contains intertwined 3D networks enabling the flow of fluids, the sinusoids for blood flow and the $\mathrm{BC}$ for bile secretion and flux ${ }^{9}$. Here, we used high resolution multiphoton microscopy and 3D digital reconstructions to generate a comparative dataset of structural changes of human liver tissue from NC, HO, STEA and eNASH patients. We identified a set of zonated morphological alterations that correlate with disease progression, such as a characteristic size distribution of LD and nuclear texture homogeneity, that can be used as tissue biomarkers to distinguish between different stages of NAFLD progression. In addition, the 3D digital reconstruction provided the first evidence that $B C$ integrity is disrupted during NAFLD progression, bringing $B C$ integrity and the mechanisms involved in its maintenance and homeostasis (cell polarity, trafficking, bile flow, BAs turnover, etc.) into focus for NAFLD studies. Based on the geometrical and topological information extracted from the BC, we used a computational personalised model to connect the microanatomy of $\mathrm{BC}$ with biliary fluid dynamics within the lobule. Our model predicted high bile pressure in the pericentral area and a significant degree of zonated cholestasis in STEA and eNASH patients, a prediction that was validated by the detection of cholestatic biomarkers in serum. Our data show that geometrical models of human tissues coupled to computational modelling is a powerful strategy to describe human physiology and physiopathology. 
315 samples revealed a set of unknown morphological features, ranging from the (sub)cellular

316 (nuclear texture, LD content, polarity) to the tissue level (BC integrity), that are perturbed

317 during NAFLD progression. First, we detected changes in nuclear texture, which have been

318 reported in several diseases $24,25,49$, but have not yet been studied in NAFLD. Indeed,

319 changes in nuclear texture homogeneity in the pericentral hepatocytes may reflect changes

320 in transcriptional activity ${ }^{27,28}$ and could serve as a new component of the histological scores

321 of NAFLD progression. Second, although the accumulation of LD is a characteristic feature

322 of NAFLD, our analysis revealed quantitative changes in their size distribution, with the

323 medium LD mostly present in eNASH and spanning the entire liver lobule. In healthy

324 conditions, the LD number and size are accurately regulated 50 and changes in LD

325 distribution point at specific alterations in the mechanisms regulating LD biogenesis and

326 catabolism. Third, and most striking, we observed alterations of the apical plasma

327 membrane of hepatocytes and of the BC network. The pericentral hepatocytes showed

328 mislocalization of DPPIV, pointing towards a dysregulation in apical protein trafficking ${ }^{37}$.

329 Interestingly, not all apical proteins were missorted, suggesting that trafficking defects could

330 be pathway- (transcytosis) and/or cargo-specific. Such defects in protein trafficking

331 correlated with the reduction of $\mathrm{BC}$ connectivity in the pericentral zone. This is the first time

332 that geometrical and topological properties of the BC are studied in human liver tissue from

333 NAFLD biopsies. The unaltered architecture of the sinusoidal network along the different

334 stages of NAFLD rules out the possibility that this reduction in BC connectivity is simply due

335 to the spatial constrains imposed by the presence of the large pericentral hepatocytes.

336 These results pose the question of how the alterations in apical surface of the hepatocytes

337 collectively result in the compromised connectivity of the $\mathrm{BC}$ network.

338 The altered BC microanatomy and the consequent increase in bile pressure in the

339 pericentral zone suggest that STEA and eNASH livers are affected by a pericentral

340 cholestasis which may contribute to the changes in BA composition observed in

341 NAFLD ${ }^{42,44,51}$. Unimpaired bile flow is essential for normal liver function. Previous studies

342 have documented that bile accumulation, due to its detergent-like properties, can cause liver

343 damage s2,53 $^{5}$ and bile pressure can affect metabolism ${ }^{54}$. The occurrence of zonated

344 cholestasis is a new piece in the NAFLD physiopathology puzzle that contributes to clarify

345 some aspects of the disease so far without explanation, e.g. increase of GGT levels ${ }^{55}$, bile

346 acids in serum ${ }^{56,57}$, upregulation of MRP3 in $\mathrm{NASH}^{58,59}$ and the beneficial effect of UDCA

347 treatment in NAFLD ${ }^{60}$, all sign of an ongoing cholestasis ${ }^{40,61,62}$. 
In recent years, a lot of research has been devoted to the role of BAs and the activity

349 of their receptor FXR, in NAFLD42-44. However, there is currently no explanation for the

350 alterations in BAs composition in blood, the decreased ratio of secondary/primary BAs

351 observed by us (Extended Data Fig. 7) and others ${ }^{42,44,51}$, and whether it correlates with

352 changes in tissue morphology ${ }^{44}$. Our data shed new light on this problem. Our results

353 suggest that the altered BC microanatomy leading to increased bile pressure in the

354 pericentral zone may hamper the ongoing bile acid secretion into BC, as apical pumps

355 (BSEP, MRP2) have to operate against elevated luminal BA concentrations (Extended Data

356 Fig. 9). This could lead to back-flux of primary BA into the blood (Extended Data Fig. 8d),

357 reducing the availability of primary bile acids to be converted into secondary bile acids by

358 the microbiota in the intestine (Extended Data Fig. 7e).

359 The combination of experimental data with computational models of tissues has

360 proven successful in elucidating pathogenetic mechanisms using animal models ${ }^{16,63,64}$.

361 However, animal models very often fail to mimic human diseases ${ }^{65}$, including NAFLD ${ }^{66}$. In

362 this study, the geometrical models of liver tissue from human biopsies combined with

363 spatially-resolved computational simulations revealed new aspects of NAFLD pathology.

364 This approach may help to identify biomarkers for early disease diagnosis and predict the

365 functional status of the tissue with potential applications in high-definition medicine ${ }^{1,67,68}$. 


\section{Acknowledgements}

368 We are grateful to Oleksandr Ostrenko, Juan Francisco Miquel Poblete and Sophie Nehring

369 for fruitful discussions, and Sebastian Bundschuh for helping to set up the 2-photon

370 microscope. We thank the Center for Information Services and High Performance

371 Computing $(\mathrm{ZIH})$ of the TU Dresden for the generous provision of computing power. We

372 would also like to thank the following Services and Facilities of the Max Planck Institute of

373 Molecular Cell Biology and Genetics for their support: Light Microscopy Facility (LMF) and

374 the Electron Microscopy Facility.

375 This work was financially supported by the German Federal Ministry of Education and

376 Research (BMBF) (LiSyM: grant \#031L0038 to M.Z., grant \#031L0033 to L.B., grant

377 \#031L0031 to J.H., DYNAFLOW: grant \#031L0082B to M.Z., grant \#031L008A to L.B. and

378 SYSBIO II: grant \#031L0044 to M.Z.), European Research Council (ERC) (grant \#695646

379 to M.Z.) and the Max Planck Society (MPG).

380 Author contributions

381 F.S-M., J.H. and M.Z. conceived the project. F.S-M., V.M. and S.S. performed the

382 immunofluorescence experiments and imaging. H.M-N. and Y.K. developed the image 383 analysis algorithms. F.S-M., V.M. and H.M-N performed the 3D tissue reconstructions. H.M384 N. and F.S-M. performed the data analysis and interpretation of the results. U.R. performed 385 the electron microscopy. A.H., S.H., C.R., and C.S obtained the samples and characterized 386 the patients. D.L. measured bile acids. M.K., F.R., Y.K. and L.B. conceived and developed 387 the mathematical model. M.K and F.R. programmed and simulated the mathematical model 388 and performed statistical analysis. M.K. and L.B. interpreted results and wrote the model 389 description. F.S-M., H.M-N., M.K., Y.K., L.B. and M.Z. wrote the manuscript. 


\section{References}

394 1. Torkamani, A., Andersen, K. G., Steinhubl, S. R. \& MD, E. J. T. High-Definition Medicine. Cell 170, 828-843 (2017).

396 2. Mills, S. E. Histology for Pathologists. (Lippincott Williams \& Wilkins, 2012).

397 3. Nalbantoglu, I. L. K. \& Brunt, E. M. Role of liver biopsy in nonalcoholic fatty liver

398 disease. World J. Gastroenterol. 20, 9026-9037 (2014).

399 4. Tanaka, N. et al. Whole-tissue biopsy phenotyping of three-dimensional tumours

$400 \quad$ reveals patterns of cancer heterogeneity. Nature Biomedical Engineering 1-14

401 (2017). doi:10.1038/s41551-017-0139-0

402 5. Hägerling, R. et al. VIPAR, a quantitative approach to 3D histopathology applied to

403

404 lymphatic malformations. JCl Insight 2, 1-14 (2017).

6. Nojima, S. et al. CUBIC pathology: three- dimensional imaging for pathological diagnosis. Sci Rep 1-14 (2017). doi:10.1038/s41598-017-09117-0

7. Glaser, A. K. et al. Light-sheet microscopy for slide-free non-destructive pathology of large clinical specimens. Nature Biomedical Engineering 1, 0084 (2017).

8. Morales-Navarrete, $\mathrm{H}$. et al. A versatile pipeline for the multi-scale digital reconstruction and quantitative analysis of 3D tissue architecture. eLife 4, 841 (2015).

9. Elias, H. A re-examination of the structure of the mammalian liver; the hepatic lobule and its relation to the vascular and biliary systems. Am. J. Anat. 85, 379-456- $15 \mathrm{pl}$ (1949).

10. Elias, H. LIVER MORPHOLOGY. Biological Reviews 30, 263-310 (1955).

11. Morales-Navarrete, $\mathrm{H}$. et al. Liquid-crystal organization of liver tissue. bioRxiv 495952 (2018). doi:10.1101/495952

12. Elias, H. A re-examination of the structure of the mammalian liver; parenchymal architecture. Am. J. Anat. 84, 311-333 (1949).

13. Treyer, A. \& Müsch, A. Hepatocyte Polarity. (John Wiley \& Sons, Inc., 2013). doi:10.1002/cphy.c120009

14. Tainaka, K., Kuno, A., Kubota, S. I., Murakami, T. \& Ueda, H. R. Chemical Principles in Tissue Clearing and Staining Protocols for Whole-Body Cell Profiling. Annu Rev Cell Dev Biol 32, 713-741 (2016).

15. Richardson, D. S. \& Lichtman, J. W. Clarifying Tissue Clearing. Cell 162, 246-257 (2015).

16. Meyer, K. et al. A Predictive 3D Multi-Scale Model of Biliary Fluid Dynamics in the Liver Lobule. Cell Systems 4, 277-290.e9 (2017).

17. Hardy, T., Oakley, F., Anstee, Q. M. \& Day, C. P. Nonalcoholic Fatty Liver Disease: Pathogenesis and Disease Spectrum. Annu Rev Pathol 11, 451-496 (2016).

18. Bedossa, P. Pathology of non-alcoholic fatty liver disease. Liver Int 37, 85-89 (2017).

19. Ke, M.-T., Fujimoto, S. \& Imai, T. SeeDB: a simple and morphology-preserving optical clearing agent for neuronal circuit reconstruction. Nature Neuroscience 16, 1154-1161 (2013).

20. Wang, M.-J., Chen, F., Lau, J. T. Y. \& Hu, Y.-P. Hepatocyte polyploidization and its association with pathophysiological processes. 8, e2805-7 (2017).

21. Younossi, Z. M. et al. Nonalcoholic fatty liver disease: assessment of variability in pathologic interpretations. Mod. Pathol. 11, 560-565 (1998). 
22. Levene, A. P. \& Goldin, R. D. Physiological hepatic nuclear vacuolation--how long does it persist? Histopathology 56, 426-429 (2010).

23. Hamilton, P. W. et al. Digital pathology and image analysis in tissue biomarker research. Methods 70, 59-73 (2014).

24. Bartels, P. H. et al. Chromatin texture signatures in nuclei from prostate lesions. Anal. Quant. Cytol. Histol. 20, 407-416 (1998).

25. Jørgensen, T., Yogesan, K., Tveter, K. J., Skjørten, F. \& Danielsen, H. E. Nuclear texture analysis: a new prognostic tool in metastatic prostate cancer. Cytometry 24, 277-283 (1996).

26. Doudkine, A., Macaulay, C., Poulin, N. \& Palcic, B. Nuclear texture measurements in image cytometry. Pathologica 87, 286-299 (1995).

27. Orr, J. A. \& Hamilton, P. W. Histone acetylation and chromatin pattern in cancer. A review. Anal. Quant. Cytol. Histol. 29, 17-31 (2007).

28. Ricci, M. A., Cosma, M. P. \& Lakadamyali, M. Super resolution imaging of chromatin in pluripotency, differentiation, and reprogramming. Curr. Opin. Genet. Dev. 46, 186-193 (2017).

29. Felmlee, D. J., Grün, D. \& Baumert, T. F. Zooming in on liver zonation. Hepatology 67, 784-787 (2018).

30. Gebhardt, R. \& Matz-Soja, M. Liver zonation: Novel aspects of its regulation and its impact on homeostasis. World J. Gastroenterol. 20, 8491-8504 (2014).

31. Kleiner, D. E. \& Makhlouf, H. R. Histology of Nonalcoholic Fatty Liver Disease and Nonalcoholic Steatohepatitis in Adults and Children. Clinics in Liver Disease 20, 293-312 (2016).

32. Kochan, K. et al. Raman spectroscopy analysis of lipid droplets content, distribution and saturation level in Non-Alcoholic Fatty Liver Disease in mice. J. Biophotonics 8, 597-609 (2015).

33. Wang, L. \& Boyer, J. L. The maintenance and generation of membrane polarity in hepatocytes. Hepatology 39, 892-899 (2004).

34. Zeigerer, A. et al. Rab5 is necessary for the biogenesis of the endolysosomal system in vivo. Nature 485, 465-470 (2012).

35. Wakabayashi, Y., Dutt, P., Lippincott-Schwartz, J. \& Arias, I. M. Rab11a and myosin $\mathrm{Vb}$ are required for bile canalicular formation in WIF-B9 cells. Proc Natl Acad Sci USA 102, 15087-15092 (2005).

36. Kipp, H., Pichetshote, N. \& Arias, I. M. Transporters on demand: intrahepatic pools of canalicular ATP binding cassette transporters in rat liver. J Biol Chem 276, 72187224 (2001).

37. Dzierlenga, A. L. \& Cherrington, N. J. Misregulation of membrane trafficking processes in human nonalcoholic steatohepatitis. J Biochem Mol Toxicol 37, e22035-7 (2018).

38. Weis, E. E. \& Barth, C. A. The extracorporeal bile duct: a new model for determination of bile flow and bile composition in the intact rat. J. Lipid Res. 19, 856-862 (1978).

39. Jansen, P. L. M. et al. The ascending pathophysiology of cholestatic liver disease. Hepatology 65, 722-738 (2017).

40. Pollock, G. \& Minuk, G. Y. Diagnostic considerations for cholestatic liver disease. J Gastroenterol Hepatol 32, 1303-1309 (2017).

41. Friedman, S. L., Neuschwander-Tetri, B. A., Rinella, M. \& Sanyal, A. J. Mechanisms of NAFLD development and therapeutic strategies. Nat Med 24, 908-922 (2018). 
505

506

507

508

509

510

511

512

513

514

515

516

517

518

519

520

521

522

523

524

525

526

527

528

529

530

531

532

533

534

42. Chow, M. D., Lee, Y.-H. \& Guo, G. L. The role of bile acids in nonalcoholic fatty liver disease and nonalcoholic steatohepatitis. Molecular Aspects of Medicine 1-11 (2017). doi:10.1016/j.mam.2017.04.004

43. Arab, J. P., Karpen, S. J., Dawson, P. A., Arrese, M. \& Trauner, M. Bile acids and nonalcoholic fatty liver disease: Molecular insights and therapeutic perspectives. Hepatology 65, 350-362 (2016).

44. Jahn, D. \& Geier, A. Bile acids in NASH: pathophysiological driving force or innocent bystanders? Hepatology 1-8 (2017). doi:10.1002/hep.29543

45. Henao-Mejia, J. et al. Inflammasome-mediated dysbiosis regulates progression of NAFLD and obesity. Nature 482, 179-185 (2012).

46. Betrapally, N. S., Gillevet, P. M. \& Bajaj, J. S. Changes in the Intestinal Microbiome and Alcoholic and Nonalcoholic Liver Diseases: Causes or Effects?

Gastroenterology 150, 1745-1755.e3 (2016).

47. Perla, F. M., Prelati, M., Lavorato, M., Visicchio, D. \& Anania, C. The Role of Lipid and Lipoprotein Metabolism in Non-Alcoholic Fatty Liver Disease. Children (Basel) 4, 46 (2017).

48. Romeo, S. et al. Genetic variation in PNPLA3 confers susceptibility to nonalcoholic fatty liver disease. Nat Genet 40, 1461-1465 (2008).

49. Huisman, A. et al. Discrimination between benign and malignant prostate tissue using chromatin texture analysis in 3-D by confocal laser scanning microscopy. Prostate 67, 248-254 (2007).

50. Walther, T. C., Chung, J. \& Farese, R. V., Jr. Lipid Droplet Biogenesis. Annu Rev Cell Dev Biol 33, 491-510 (2017).

51. Puri, P. et al. The presence and severity of nonalcoholic steatohepatitis is associated with specific changes in circulating bile acids. Hepatology 67, 534-548 (2017).

52. Trauner, M., Meier, P. J. \& Boyer, J. L. Molecular pathogenesis of cholestasis. N Engl J Med 339, 1217-1227 (1998).

53. Coleman, R., lqbal, S., Godfrey, P. P. \& Billington, D. Membranes and bile formation. Composition of several mammalian biles and their membrane-damaging properties. Biochem J 178, 201-208 (1979).

54. Jansen, P. L. M. Hydrodynamics of bile flow: Lessons from computational modeling. Hepatology 67, 1624-1627 (2018).

55. Chan, T. T. \& Wong, V. W. S. In search of new biomarkers for nonalcoholic fatty liver disease. Clinical Liver Disease 8, 19-23 (2016).

56. Ferslew, B. C. et al. Altered Bile Acid Metabolome in Patients with Nonalcoholic Steatohepatitis. Digestive Diseases and Sciences 60, 3318-3328 (2015).

57. Mouzaki, M. et al. Bile Acids and Dysbiosis in Non-Alcoholic Fatty Liver Disease. PLOS ONE 11, e0151829-13 (2016).

58. Canet, M. J. et al. Altered Regulation of Hepatic Efflux Transporters Disrupts Acetaminophen Disposition in Pediatric Nonalcoholic Steatohepatitis. Drug Metab Dispos 43, 829-835 (2015).

59. Hardwick, R. N., Fisher, C. D., Canet, M. J., Scheffer, G. L. \& Cherrington, N. J. Variations in ATP-binding cassette transporter regulation during the progression of human nonalcoholic fatty liver disease. Drug Metab Dispos 39, 2395-2402 (2011).

60. Steinacher, D., Claudel, T. \& Trauner, M. Therapeutic Mechanisms of Bile Acids and Nor-Ursodeoxycholic Acid in Non-Alcoholic Fatty Liver Disease. Dig Dis 35, 282-287 (2017). 
61. Chai, J. et al. Elevated hepatic multidrug resistance-associated protein 3/ATPbinding cassette subfamily $\mathrm{C} 3$ expression in human obstructive cholestasis is mediated through tumor necrosis factor alpha and c-Jun $\mathrm{NH} 2$-terminal kinase/stress-activated protein kinase-signaling pathway. Hepatology 55, 14851494 (2012).

62. Donner, M. G. \& Keppler, D. Up-regulation of basolateral multidrug resistance protein 3 (Mrp3) in cholestatic rat liver. Hepatology 34, 351-359 (2001).

63. Hoehme, S. et al. Model Prediction and Validation of an Order Mechanism Controlling the Spatiotemporal Phenotype of Early Hepatocellular Carcinoma. Bull Math Biol 1-38 (2018). doi:10.1007/s11538-017-0375-1

64. Menshykau, D. et al. Image-based modeling of kidney branching morphogenesis reveals GDNF-RET based Turing-type mechanism and pattern-modulating WNT11 feedback. Nature Communications 10, 239 (2019).

65. Uhl, E. W. \& Warner, N. J. Mouse Models as Predictors of Human Responses: Evolutionary Medicine. Curr Pathobiol Rep 3, 219-223 (2015).

66. Lau, J. K. C., Zhang, X. \& Yu, J. Animal models of non-alcoholic fatty liver disease: current perspectives and recent advances. J. Pathol. 241, 36-44 (2017).

67. Whitcomb, D. C. What is personalized medicine and what should it replace? Nat Rev Gastroenterol Hepatol 9, 418-424 (2012).

68. Amalou, H. \& Wood, B. J. Biopsy and personalized medicine. Nat Rev Gastroenterol Hepatol 9, 683-author reply 683 (2012). 


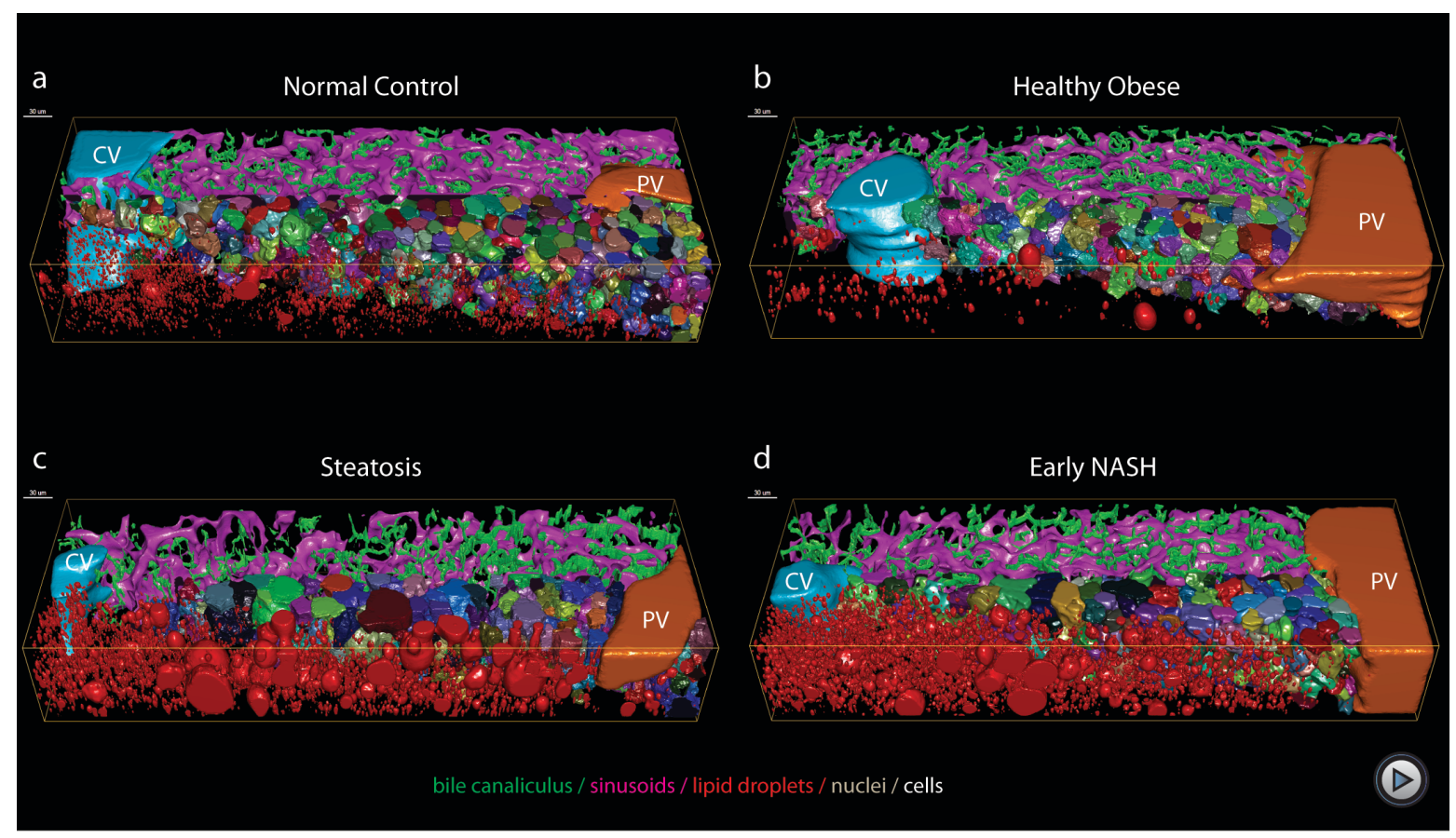

558 Fig. 1.3D reconstruction and quantitative analysis of human liver morphology. Human

559 liver sections obtained by biopsy ( 100 um thick) were stained for bile canaliculi (CD13),

560 sinusoids (fibronectin), nucleus (DAPI), lipid droplets (BODIPY) and cell border (LDLR),

561 optically cleared with SeeDB and imaged at high resolution using multiphoton microscopy

$562(0.3 \mu \mathrm{m} \times 0.3 \mu \mathrm{m} \times 0.3 \mu \mathrm{m}$ per voxel). For each sample, we reconstructed the central vein

563 (light blue), portal vein (orange), bile canaliculus (green), sinusoids (magenta), lipid droplets

564 (red), nuclei (random colours) and hepatocytes (random colours). a, Normal control. b,

565 Healthy obese. c, Steatosis. d, Early NASH. 
a

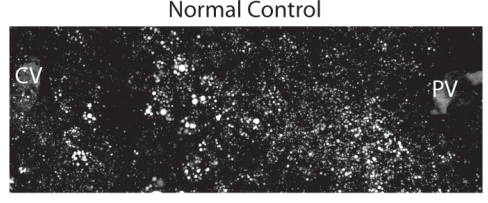

Steatosis
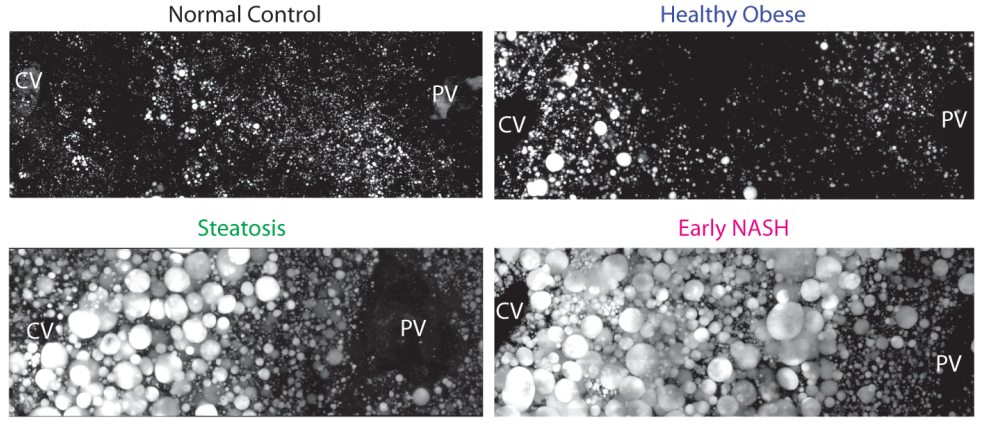

Early NASH
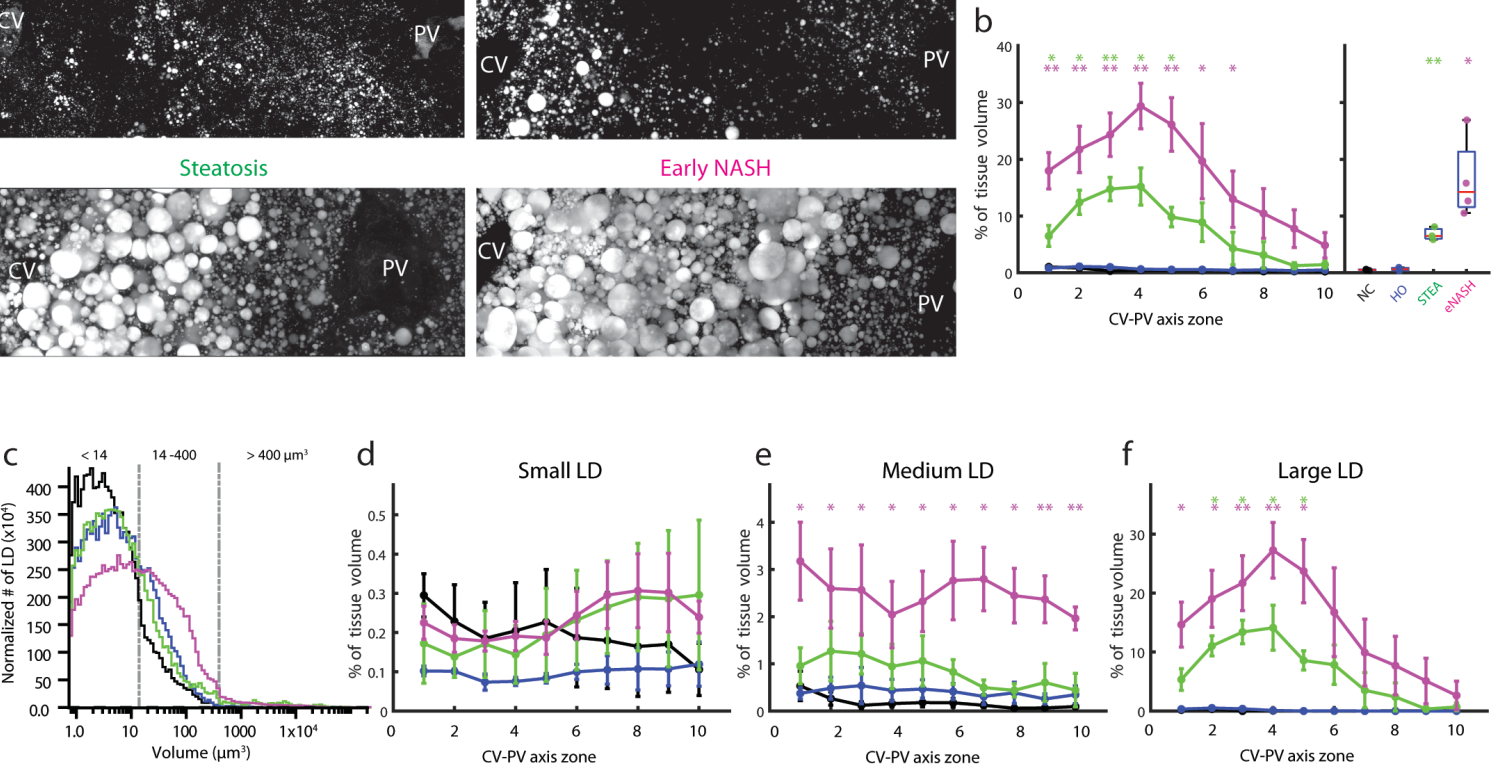

Fig. 2. Quantitative characterization of LD along the CV-PV axis. a, Representative IF images of fixed human liver tissue sections stained with BODIPY. Shown is a maximum projection of a $60 \mu \mathrm{m}$ z-stack covering an entire CV-PV axis. b, Quantification of the percentage of tissue volume occupied by the LD along the CV-PV axis and the overall values (i.e. over the whole CV-PV axis). c, LD volume distribution. LD populations were defined based on the LD volume distribution of the NC group (black line). Three populations of LD were defined based on their volume: small $\left(<14 \mu \mathrm{m}^{3}\right)$, medium $\left(14-400 \mu \mathrm{m}^{3}\right)$ and large $(>$

$574400 \mu^{3}$ ), corresponding to changes in the volume distribution (i.e. drops in the number of

575 LD). Quantification of the percentage of tissue volume occupied by the LD along the CV-PV

576 for (d) small, (e) medium and (f) large LD. NC $=3$ samples, HO = 3 samples, STEA = 3

577 samples, eNASH $=3$ samples. Spatially-resolved quantification represented by mean \pm SEM

578 per zone and overall quantifications by box-plots. ${ }^{*} p$-values $<0.05,{ }^{* *} p$-values $<0.01,{ }^{* * *} p-$

579 values $<0.001$ 


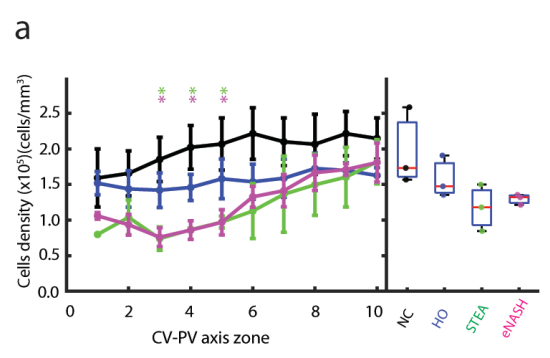

d

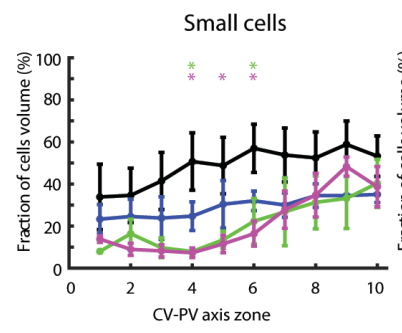

h

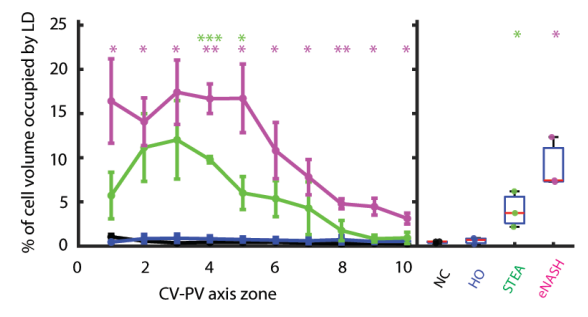

b

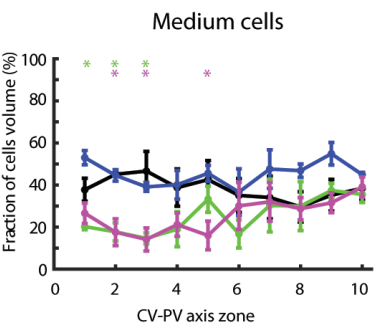

f
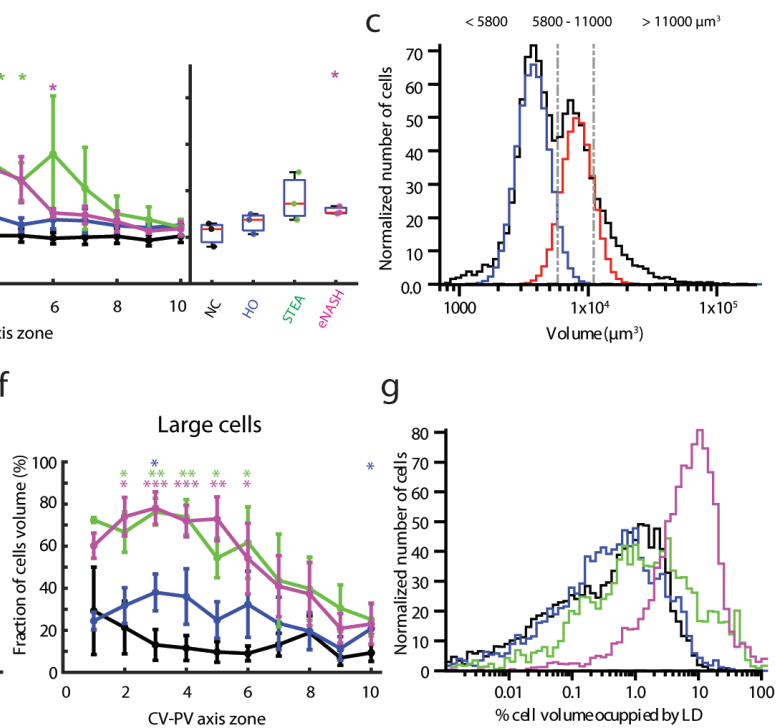

9
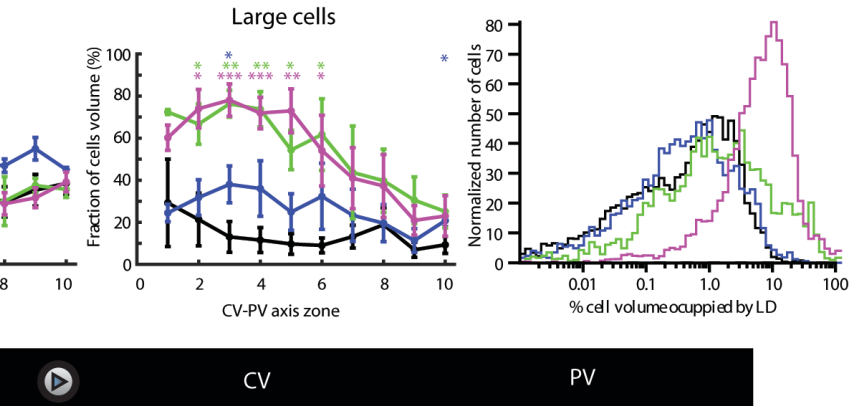

C C

PV

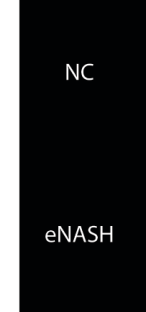

Figure 3. Cell based analysis of NAFLD. Quantification of the number of hepatocytes per tissue volume unit (a) and cell volume (b) both, along the liver lobule and the overall average.

583 c, Cell volume distribution. For the population analysis, the hepatocytes form all the groups were pulled together and the populations were defined based on their volume distribution (c). By fitting the volume distribution with two normal distributions (c), the volume values defining three population's boundaries were identified: small $\left(<5800 \mu \mathrm{m}^{3}\right)(\mathbf{d})$, medium $587\left(5800-11000 \mu \mathrm{m}^{3}\right)(\mathbf{e})$ and large $\left(>11000 \mu \mathrm{m}^{3}\right)(\mathbf{f})$. The fraction of cellular volume occupied 588 by the different populations is shown in $\mathbf{d}, \mathbf{e}$, and $\mathbf{f}$. Percentage of the cell volume occupied by lipid droplets: distribution (g) and statistics along the CV-PV axis and overall (h). NC = 3 590 samples, $\mathrm{HO}=3$ samples, STEA $=3$ samples, eNASH $=3$ samples. Spatially-resolved 591 quantification represented by mean \pm SEM per zone and overall quantifications by box-plots. $592{ }^{*} \mathrm{p}$-values $<0.05,{ }^{* *} \mathrm{p}$-values $<0.01,{ }^{* *} \mathrm{p}$-values $<0.001$. Representative cells reconstructed 593 in 3D and selected from zone 3 and 8 . Apical, basal and lateral surface are shown in green, 594 magenta and grey respectively. Lipid droplets are shown in red. Scale bar, $10 \mu \mathrm{m}$. 


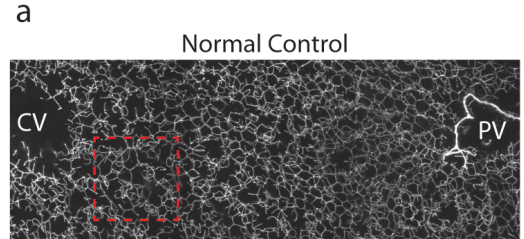

Steatosis

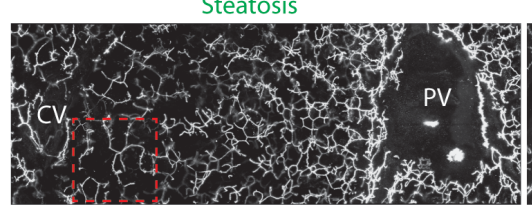

C

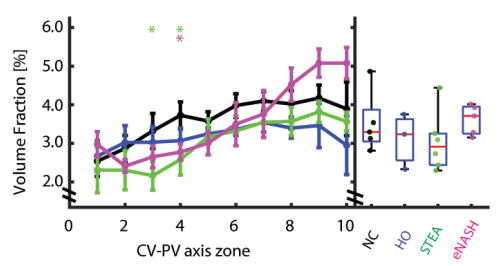

$\mathrm{f}$

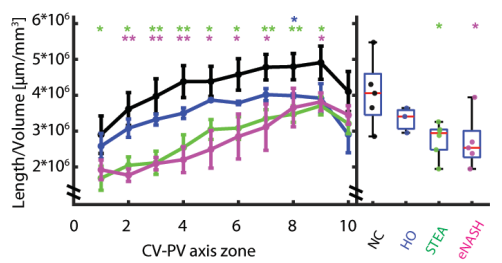

d

9
Healthy Obese

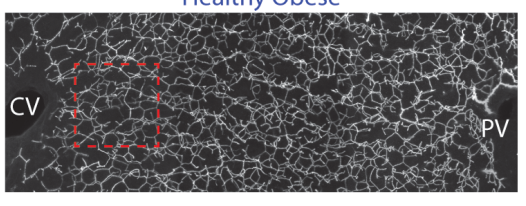

Early NASH

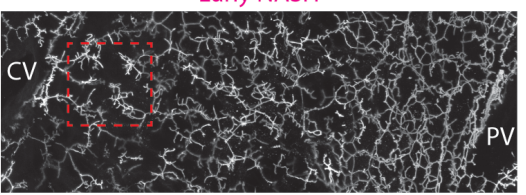

b

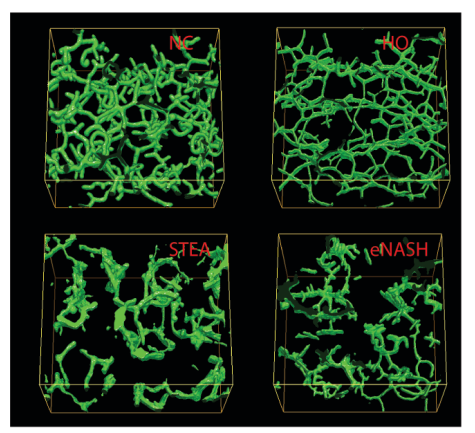

e

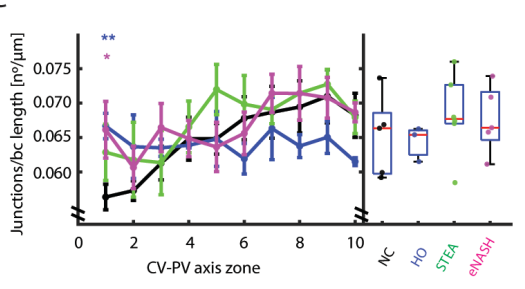

h

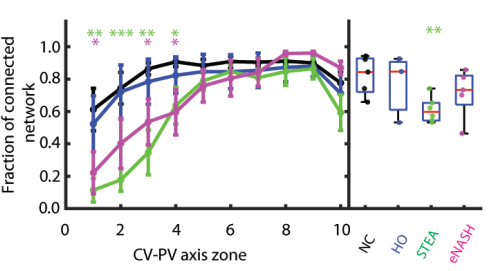

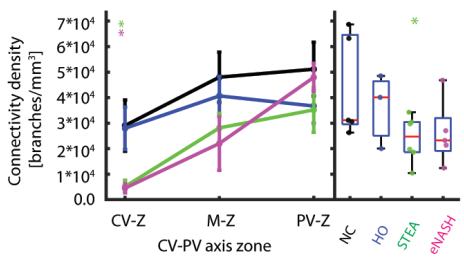

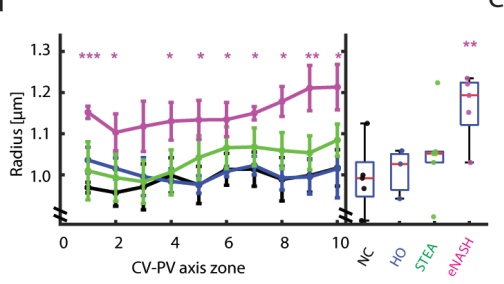

h

596 Figure 4. Structural and topological defects of bile canaliculi revealed by spatial 3D

597 analysis. a, Representative IF images of fixed human liver tissue sections stained with

598 CD13 after citric acid antigen retrieval. Shown is a maximum projection of a $60 \mu \mathrm{m}$ z-stack

599 covering an entire CV-PV axis. b, Inset showing 3D representation of the bile canaliculus

600 highlighted in a. Quantification of the volume fraction of tissue occupied by bile canaliculi(c),

601 radius (d), number of junctions (e), total length per volume (f), fraction of connected network

602 (g) and connectivity density (h) of the BC network along the CV-PV axis and overall (See

603 Methods for details). $\mathrm{NC}=5$ samples, $\mathrm{HO}=3$ samples, STEA $=6$ samples, eNASH $=5$

604 samples. Spatially-resolved quantification represented by mean \pm SEM per zone and overall

605 quantifications by box-plots. ${ }^{*} p$-values $<0.05$, ${ }^{* *} p$-values $<0.01$, ${ }^{* * *} p$-values $<0.001$. 
a

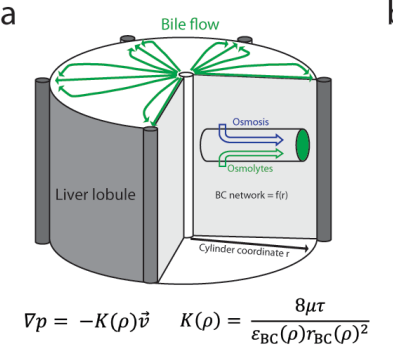

d

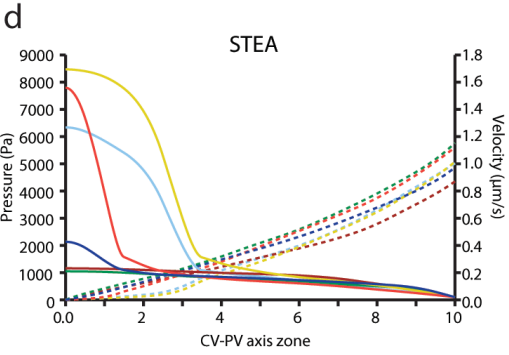

b

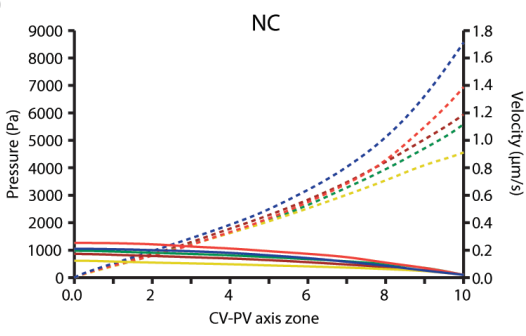

e
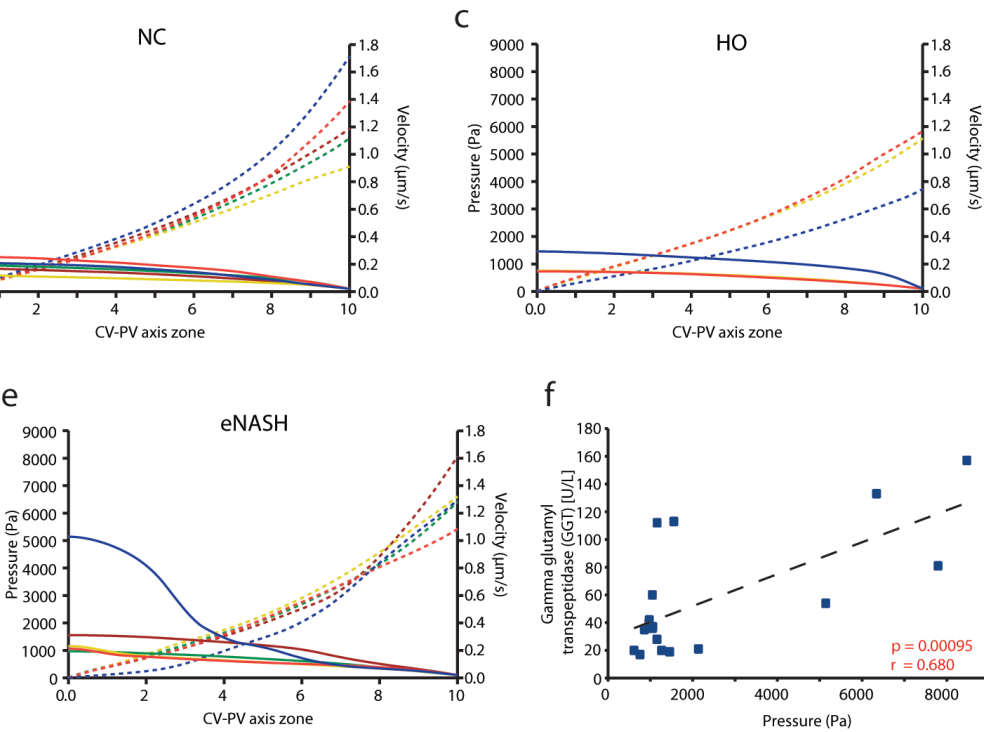

$f$

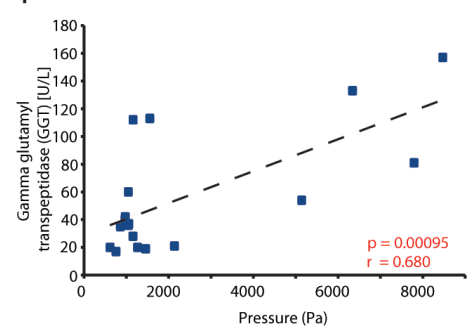

Figure 5. Individual-based model prediction of bile pressure $p$ and flow velocity $\vec{v}$ profiles based on measured bile canalicular geometries. a, Abstraction of liver lobule by

609 cylinder symmetry with radial coordinate $\rho$. The mechanistic model considers secretion of 610 osmolytes (green) and osmotic water influx (blue) in a porous medium with $\rho$-dependent 611 properties (see supplemental model description). Darcy's law is assumed with a 612 proportionality constant $\mathrm{K}(\rho)$ depending on viscosity $\mu$, tortuosity $\tau$, bile canalicular volume 613 fraction $\varepsilon_{\mathrm{BC}}$, bile canalicular radius $r_{\mathrm{BC}}$. All geometric parameters have been measured per 614 patient. b-e, Model prediction for bile fluid pressure (solid line, left axis) and bile flow velocity 615 (dashed line, right axis) profiles for individual patients (colour) in for disease groups. NC $=5$ 616 samples, $\mathrm{HO}=3$ samples, STEA $=6$ samples, eNASH $=5$ samples. $\mathrm{f}$, Scatter plot of 617 measured Gamma glutamyl transpeptidase (GGT) levels versus predicted pericentral (zone 618 0) bile fluid pressure from individual patients from all groups reveals a statistically significant 619 positive correlation. One-sided t-test. P-values and Pearson correlation coefficient are 620 indicated in the plots. 